\title{
Trans-catheter closure of aortic anastomosis leak after aortic replacement: classifications and techniques
}

\author{
Wenhui $\mathrm{Wu}^{1}$, Yutong $\mathrm{Ke}^{2}$, Honglei Zhao ${ }^{3}$, Lianjun Huang ${ }^{1}$, Junzhou $\mathrm{Pu}^{1}$ \\ ${ }^{1}$ Interventional Department, ${ }^{2}$ Echocardiography Department, ${ }^{3}$ Cardiology Surgery Department, Beijing Anzhen Hospital, Capital Medical \\ University, Beijing, China \\ Contributions: (I) Conception and design: W Wu, J Pu; (II) Administrative support: W Wu, L Huang, J Pu; (III) Provision of study materials or \\ patients: All authors; (IV) Collection and assembly of data: W Wu, Y Ke, J Pu; (V) Data analysis and interpretation: W Wu, Y Ke, H Zhao, J Pu; (VI) \\ Manuscript writing: All authors; (VII) Final approval of manuscript: All authors. \\ Correspondence to: Junzhou Pu, MD. Beijing Anzhen Hospital, No.2 Anzhen Road, Beijing, China. Email: junzhoupu@163.com.
}

\begin{abstract}
Background: Aortic anastomotic leak (AAL) is knotty complication after aortic replacement. We aimed to evaluate the feasibility and efficacy of the techniques of trans-catheter AAL closure as well as to evaluate the impact of the new classification on the interventional closure.

Methods: From October 2015 to November 2017, 20 consecutive high surgical risk patients (mean age $47 \pm 12$ years, 13 males) were referred to our center for trans-catheter closure AALs. Due to the variation of leak, we therefore developed a new-classification based on transesophageal echocardiography (TEE) and computed tomography angiography (CTA) assessments: type I: aorta-to-right atrium fistula, n=6; type II: pseudoaneurysm induced by a suture line dehiscence, $n=4$; type III: patency of the false lumen in aortic dissection, $\mathrm{n}=10$. Outcomes were analyzed by assessing TEE and CTA in different types of AALs.

Results: Successful closure was accomplished in 17 subjects (85\%). The severity of AAL reduced significantly in 15 patients (88\%); two patients required a second procedure. At follow-up, we found that in type $\mathrm{I}$, the right atrium systolic pressure reduced (from $25.3 \pm 4.1$ to $7.0 \pm 1.2 \mathrm{mmHg}$ ) with the improved NYHA $(3.5 \pm 0.6$ vs. $1.0 \pm 0.0)$, the diameter of pseudoaneurysm significantly decreased $(5.0 \pm 1.8$ to $2.0 \pm 1.8 \mathrm{~mm}$ ) in type II, and complete thrombosis was achieved in all type III patients.

Conclusions: Trans-catheter closure of AAL displays satisfactory results even in those defined as high-risk patients, and it could be considered be a viable alternative approach. New classification is helpful in decisionmaking.
\end{abstract}

Keywords: Aortic surgery; complication; anastomotic leak; trans-catheter closure

Submitted Mar 17, 2020. Accepted for publication Jul 18, 2020.

doi: $10.21037 /$ jtd-20-1496

View this article at: http://dx.doi.org/10.21037/jtd-20-1496

\section{Introduction}

Aortic anastomotic leak (AAL) is not uncommon with a reported incidence of $5-8 \%(1,2)$. The main risk factors of AAL include calcification or degeneration of the aortic wall, surgical procedure type, unqualified surgical technique, infection, and underlying Marfan syndrome $(3,4)$. Moreover, it may be observed when a suture line dehiscence occurs between the aortic prosthesis and the native aorta after surgical aortic replacement.
AAL can lead to pseudoaneurysm, patency of false lumen or even aortic rupture and is associated with a high mortality and morbidity (5-7). Surgical repair of AAL is considered as the standard therapy; however, the mortality of redo-surgery is relatively high ranging from $13-41 \%$, sometimes even worse than primary aortic surgery (8-11). Recently, interventional AAL closure has emerged as an alternative approach in a few anecdotal case reports or small series of selected patients (12-14). Due to the significant heterogeneous clinical phenotypes of AAL, the clear 
classification is of importance for interventional decisionmaking. But currently, there is lack of the classification especially targeting for trans-catheter closure. We therefore conducted this study to define the clinical classification and to assess the feasibility and efficacy of the trans-catheter closure of in one single-center with consecutive case series We present the following article in accordance with the STROBE reporting checklist (available at http://dx.doi. org/10.21037/jtd-20-1496).

\section{Methods}

\section{Patients' enrollment}

The study was conducted in accordance with the Declaration of Helsinki (as revised in 2013). The study was approved by institutional ethics board of Beijing Anzhen Hospital, and informed consent was taken from all the patients. We retrospectively identified 20 consecutive patients who underwent percutaneous intervention for AALs at our center between October 2015 and November 2017. The Society of Thoracic Surgeons risk score and the EuroSCORE II system are widely used for surgical risk evaluation in cardiac surgery; however, such scores have been validated only in standard surgical-risk patients, and they may fail to adequately capture risk factors for patients undergoing AAL closure. These factors must be considered by the heart team when deciding on the appropriateness of intervening. The indications for percutaneous in our center were patients in high risk of redo-surgery after assessment by our heart team, as well as meeting one of the conditions: (I) with severe dyspnea; (II) asymptomatic but with moderate or severe AAL; (III) with persistent bleeding evidenced by medical imaging and have the risk for rupture of the pseudoaneurysm or the false lumen. The patients who were with a history of Behcet's syndrome or couldn't tolerate general anesthesia was defined as in high risk of redosurgery. Percutaneous repair is contraindicated in patients with active endocarditis or significant dehiscence involving more than one-fourth to one-third of the aortic prosthesis.

\section{Definitions of terminology}

\section{Classification}

AAL is defined as a shunt originating from the aortic lumen through the suture dehiscence between the aortic prosthesis and the native aorta. Diagnosis of AAL was based on transesophageal echocardiography (TEE) and computed tomography angiography (CTA) assessments. According to the hemodynamic and the leak location, AALs were classified into 3 types (Figure 1). Type I AAL indicates aorta-to-right atrium fistula and patency of false lumen in the ascending aorta after a Cabrol-shunt-technique was performed, which aims to connect right atrium and aortic wall to decompress the peri-aortic space, thus can autotransfuse the shed blood due to an intractable hemorrhage after aortic reconstruction (2) (Figure 1A). Type II AAL means a localized hematoma (pseudoaneurysm), whereby a suture line dehiscence occurs at the proximal or distal suture lines (Figure 1B). Type III AAL is the continued patency of the false lumen, leading to procedural ineffectiveness of the primary surgery for the aortic dissection (Figure 1C). For the lesion location, type I and type II AALs frequently locate between the native ascending aorta and the aortic prosthesis after an ascending aorta replacement; and in the anastomosis of the aortic arch and the stent-graft for type III (Figure 1).

\section{Technical success}

Technical success was considered be achieved if both the following criteria met: (I) the successful implantation of occluder(s) without interfering with the aortic valve, as well as the blood flow in the aorta or major branches; (II) the shunt of the AAL reduced at least one grade. Actually, assessment of AAL in our centre can be challenging and requires an integrative approach. Echocardiographic assessment of regurgitation should consider an assessment of the AALs' type and quantification of regurgitant severity. Refer to the standard for assessment of the aortic prosthetic paravalvular leak's regurgitation, the severity of each type I or type III AAL was evaluated by 2D-TEE and RT-3DTEE using several parameters: area of the color-Doppler blood shunt area (jet area, JA), the narrowest diameter of the leak jet [vena contracta (VC)] and 3D color effective regurgitation orifice (ERO). The degree of blood shunt was classified into 3 grades: I (mild, VC: $<0.3 \mathrm{~cm}, \mathrm{JA}:<6 \mathrm{~cm}^{2}$, ERO: $<0.1 \mathrm{~cm}^{2}$ ); II (moderate, VC: $0.3-0.6 \mathrm{~cm}, \mathrm{JA}: 3-6 \mathrm{~cm}^{2}$, ERO: $0.1-0.3 \mathrm{~cm}^{2}$ ), III (severe, VC: $>0.6 \mathrm{~cm}$, JA: $>6 \mathrm{~cm}^{2}$, ERO: $>0.3 \mathrm{~cm}^{2}$ ). For type II AALs, 3D color ERO is more difficult to assess, however, the JA/pseudoaneurysm area (\%) can be used to grade severity of the regurgitation, a 3-class grading scheme have also been used to report the severity of regurgitation for type II AAL, I (mild, VC: $<0.3 \mathrm{~cm}$, JA: $<6 \mathrm{~cm}^{2}, \mathrm{JA} /$ pseudoaneurysm area: $<30 \%$ ); II (moderate, VC: $0.3-0.6 \mathrm{~cm}, \mathrm{JA}: 3-6 \mathrm{~cm}^{2}, \mathrm{JA} /$ pseudoaneurysm area: $30-45 \%$ ); III (severe, VC: $>0.6 \mathrm{~cm}, \mathrm{JA}:>6 \mathrm{~cm}^{2}, \mathrm{JA} /$ pseudoaneurysm area: $>45 \%)$. 
A

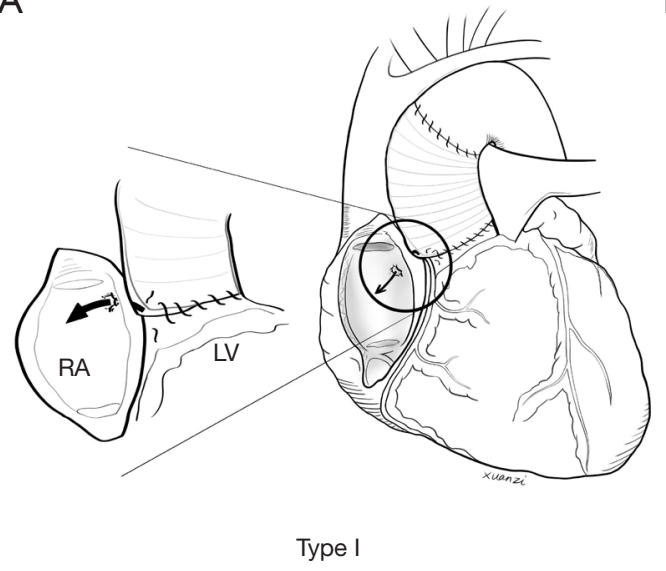

C

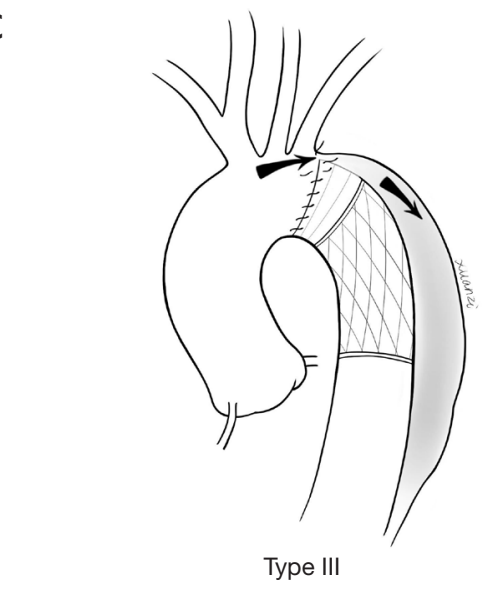

B

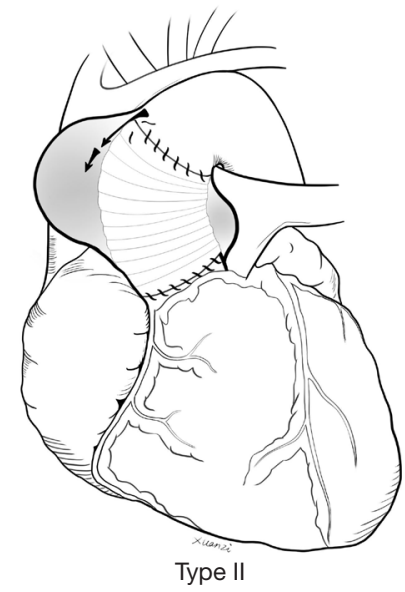

Figure 1 Illustration of the classifications of AAL. (A) The diagram of type I AAL; (B) type II AAL; (C) type III AAL. AAL, aortic anastomotic leak; RA, right atrium; LV, left ventricle.

\section{Clinical success}

Clinical success should meet the criteria at least one of the following requirements:

(I) The New York Heart Association (NYHA) function improved for at least one class in patients with a type I AAL.

(II) The diameter of the pseudoaneurysm decreased in patients with a type II AAL.

(III) Stent-segment (the frozen elephant trunk) thrombosis of the aortic false lumen was achieved in patients with a type III AAL.

\section{Multiple-modality imaging evaluation at baseline}

Prior to trans-catheter closure of the AAL, the location, shape, type and size of the AAL were assessed by TEE and aortic CTA, i.e., two-dimensional (2D)-TEE, real- time 3D-TEE, and electrocardiography (ECG)-gated 3D CTA. It was found that most of the leaks were located at the proximal anastomosis of the stent-graft [11 (55\%)] after a frozen elephant trunk technique, it less frequently located at the aortic prosthesis of the proximal site [6 (30\%) or the distal end $3(15 \%)]$. Half of the AALs were type III [10 $(50 \%)]$, the other half were type I [6 $(30 \%)]$ or type II [4 (20\%)]. Prior to trans-catheter closure of the AAL, the shunt degree was mainly severe (12 patients, $60 \%$ ), or moderate (8 patients, $40 \%$ ).

\section{Techniques for the trans-catheter closure of the $A A L$}

Trans-catheter closure was performed in the catheter lab room using local anesthesia. During the procedure, every patient received intravenous heparin with dosage of $100 \mathrm{U} / \mathrm{kg}$. Different strategies of occlusion were used according to 
AAL types. The techniques are described as follows.

\section{Type I AAL closure}

A 6-French $(6-\mathrm{F})$ access was obtained in the femoral artery and vein separately. The right atrium systolic pressure was recorded with a 6-F multipurpose catheter (Medtronic, Minneapolis, USA). Under the guidance of a 5F JR 4.0 or Cobra catheter (Medtronic, Minneapolis, USA), the arteriovenous loop was established with a $260 \mathrm{~cm}, 0.035$ inch, hydrophilic guide wire (Terumo, Tokyo, Japan) by crossing both the leak and the Cabrol's shunt and subsequently advancing into the right atrium and then snaring and exteriorizing from the femoral vein. Then the delivery sheath (6- to 8-F according to the device size) crossed the leak in retrograde approach. The occlusion device with appropriate size determined by AAL size and shape, was introduced alongside the loop, and carefully deployed across the leak. When the technical success was obtained, the loop guide wire could be was removed and the occluder was finally released (Figure $2 A, B$ ).

\section{Type II AAL}

A $260 \mathrm{~cm}, 0.035$-inch, hydrophilic guidewire approached to the leak (the entry of the aortic pseudoaneurysm) by the use of different catheters. After crossing the leak, the hydrophilic guidewire was replaced by an Amplatzer super stiff guidewire (Boston Scientific, Marlborough, USA). A delivery sheath was advanced over the super stiff guidewire. At this stage, the operator should notice the tip of the super stiff guidewire and the dilator of the sheath to prevent the aortic pseudoaneurysm puncture. Finally, the closure device was implanted at the leak (Figure 2C,D).

\section{Type III AAL}

The applied technique was like that in type I AAL as mentioned above, but the loop was oriented from aortic true lumen to the false lumen. Therefore, 2 accesses should be prepared including the regular femoral/radial artery access and a femoral artery with false lumen was involved. When a femoral artery was not involved in the false lumen, the guidewire was advanced from the true lumen into the false lumen via the distal intimal tear of the aortic dissection and was snared and crossed the leak to establish the true lumen-to-false lumen loop. The device was finally deployed at the leak (Figure 2E,F).

There is no specific device for trans-catheter AAL closure. In our series, an appropriate device should be applied to maximize the efficiency of the closure procedure.
Amplatzer vascular plug (AVP III) (AGA Medical, Plymouth, USA) was implanted considering its shape matching the morphology of the AAL, mostly in the oval or crescentic shape. Ventricle septal defect occluder (VSO) (Lifetech, Shenzhen, China) or Amplatzer duct occluder I (ADO I) (AGA Medical, Plymouth, USA), with its large size, accomplished the trans-catheter closure of large leaks. ADO II (AGA Medical, Plymouth, USA), with a small and soft Torqve LP sheath (AGA Medical, Plymouth, USA) which enabled crossing the leaks smoothly, was implanted to close small leaks.

\section{Follow-up}

The follow-up was carried out at pre-discharge, 1 month, 6 months and then every year post the procedure. During the follow-up, physical examinations, ECG, transthoracic echocardiography were done, and ECG-gated aortic CTA evaluations were performed only at 6 and 12 months after the procedure.

\section{Statistical analysis}

Continuous variables are presented using mean \pm SD or medians with interquartile range (IQR) as appropriate. Categorical variables are presented as numbers (percentages). Data was analyzed with dedicated software [SPSS: version 20.0 (IBM SPSS, Chicago, Illinois)].

\section{Results}

\section{Baseline characteristics}

The majority of the patients were males [13 (65\%)]. The mean age was $47 \pm 12$ years (range, 26-69 years). The aortic dissection was performed in 16 patients $(80 \%)$ and aortic aneurysm in 4 patients (20\%) regarding to primary aortic surgery. The median time was 15 months (IQR: 7.0-28 months) after the primary aortic surgery. Most of patients had comorbidity including primary hypertension [18 $(90 \%)]$, heart failure [10 (50\%)]. Patients were referred due to radiological detection of an AAL by CTA regardless patients asymptomatic $[10(50 \%)]$ or symptomatic [dyspnea: 10 (50\%), chest pain: 3 (15\%)]. More details are listed in Table 1.

\section{Technical outcome}

In all, occlusion devices were initial success deployed in 17 $(85 \%)$ patients with various types of devices. The applied 

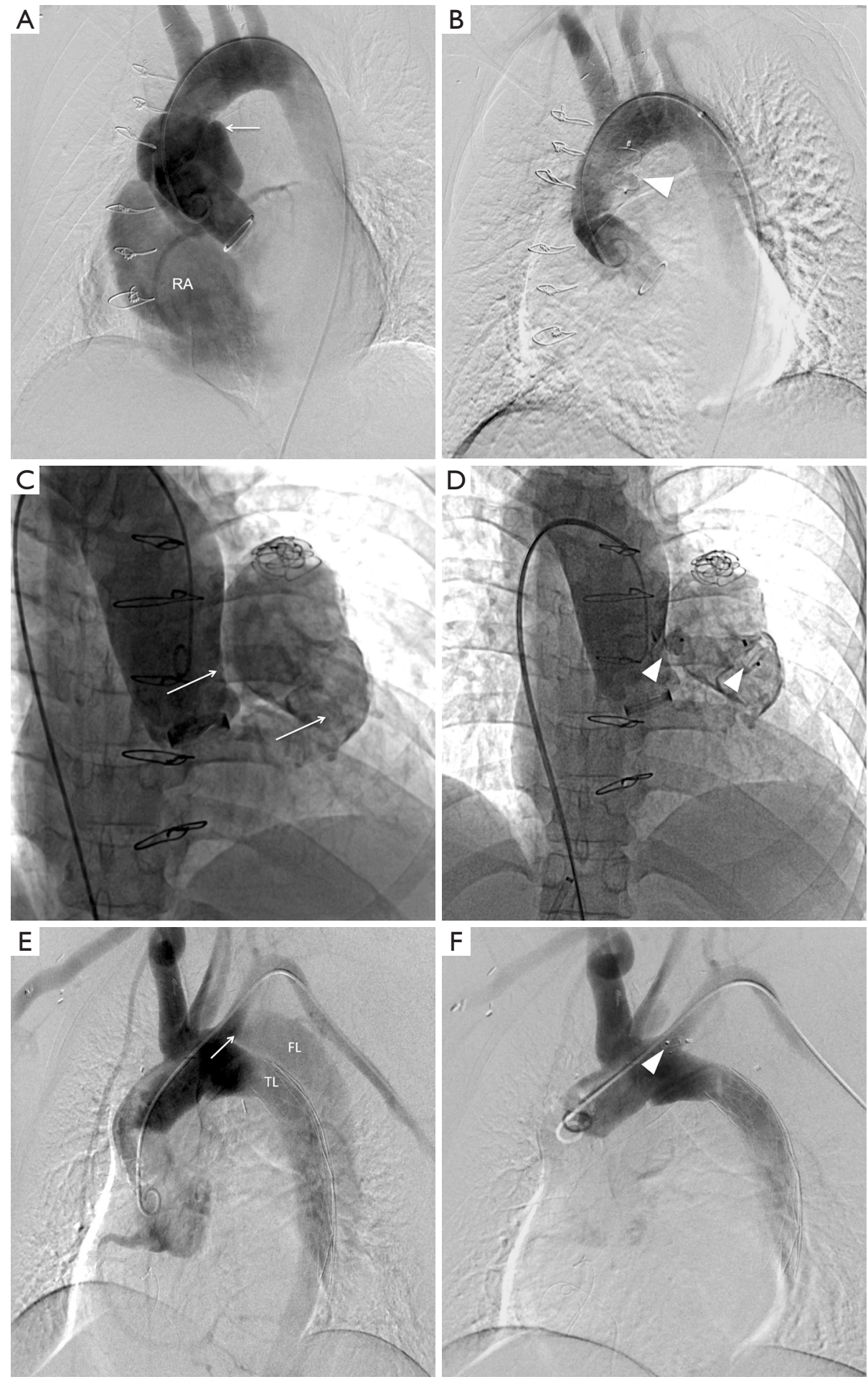

Figure 2 Procedures of different types of aortic anastomotic leak (AAL) closure. (A) Fistula (arrow) originating from the aorta and draining into the right atrium (RA) in a type I AAL; (B) the AAL was occluded by a $14 \mathrm{~mm}$ VSO (arrowhead); (C) a pseudoaneurysm caused by a type II AAL which had an entry (arrow) located at the aortic anastomosis and an exit located at the pulmonary artery (arrow); (D) both the entry and the exit were occluded by double $14 \times 5 \mathrm{~mm}$ AVP III (arrowhead); (E) type III AAL (arrow) led to false lumen (FL) patency of aortic dissection; (F) the AAL was occluded by a $14 \times 5 \mathrm{~mm}$ AVP III (arrowhead). 


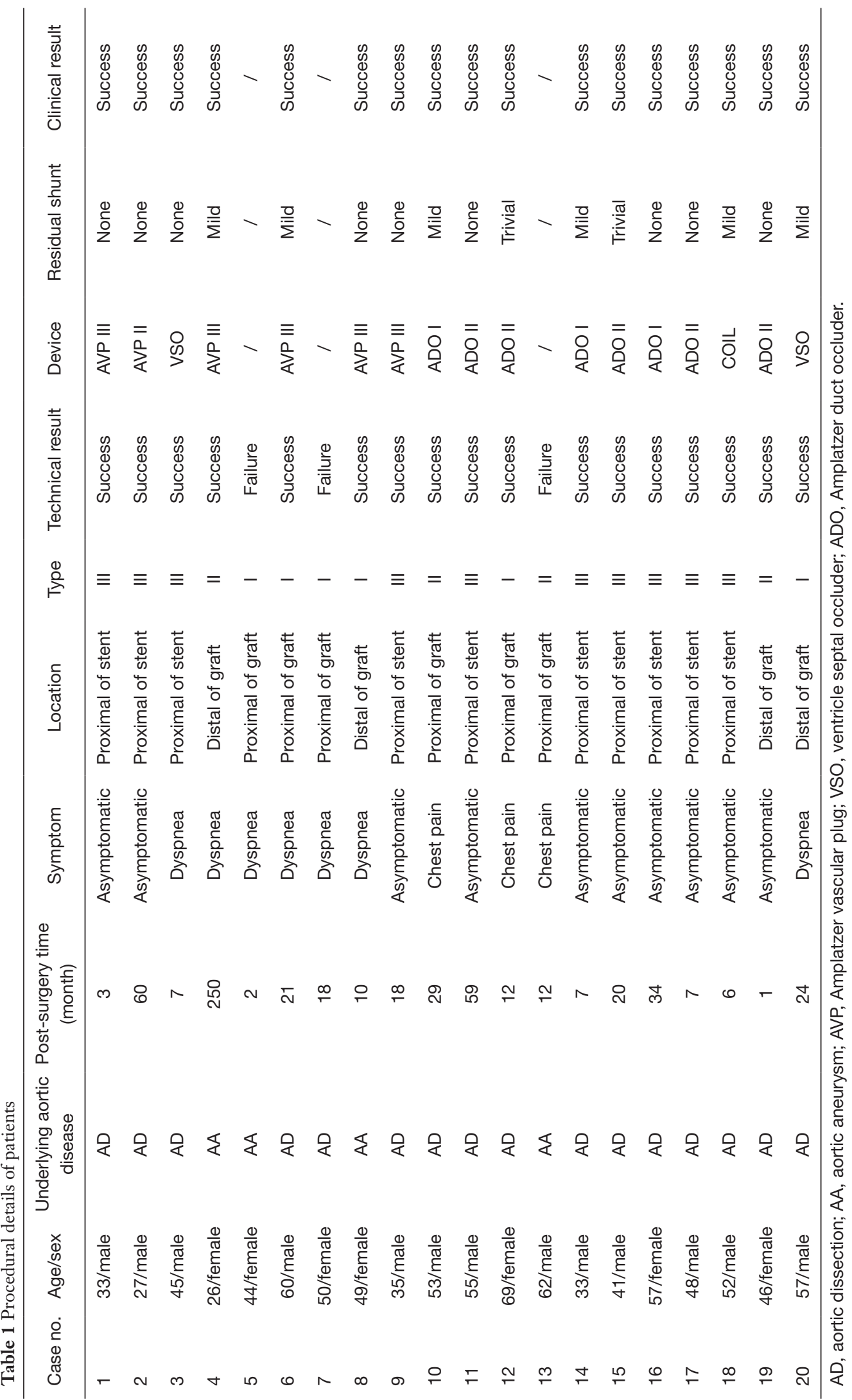


devices in this case series were $\mathrm{ADO}$ II $(7,35 \%)$, followed by $\operatorname{AVP}$ III (6, 30\%), and ADO I (3, 15\%), respectively. The remaining patients were implanted with VSO, AVP II and coil (Table 1). The overall technical successful rate was $85 \%(n=17)$ in these subjects. Of note, there was no death, procedure-related complications including myocardial infarction or stroke.

For type I AALs (6 patients), 4 closures were performed with AVP III ( $\mathrm{n}=2)$, ADO II $(\mathrm{n}=1)$ and VSO $(\mathrm{n}=1)$. However, closing procedure was unsuccessful in the other two patients since the delivery sheath could not cross the leak by either antegrade and retrograde manner over the guidewire loop.

Two AVP III, 1 ADO I and 1 ADO II were deployed in 3 patients for those defined as type II subgroup $(n=4)$. Notably, one patient had a pseudoaneurysm with an aortic entry and a pulmonary artery exit, and we thereby implanted 2 AVP III devices. Occlusion was failed in one patient; both the delivery sheath and the guiding catheter were unable to be inserted into the pseudoaneurysm over the guidewire. Redosurgery was refused to by the patient and he eventually died of pseudoaneurysm rupture 3 months later.

In the subgroup ascribed to type III AALs $(n=10)$, the loop from true lumen to false lumen was successfully established in all patients. For 1 patient, an ASD occluder migrated into the aortic false lumen after release, but snare technology was unsuccessful and an $A D O I$ was implanted at the leak immediately. Fortunately, the $A S D$ device was stable during the follow-up and further observation should be warranted over a longer period. Two patients received a planned secondary repair of the residual shunt with $A D O I I$ after first attempt using $A D O I$ and coils, respectively.

\section{Clinical outcome}

The median follow-up was 12 months (IQR: 6.0-24.0 months) and all successful 17 patients survived during follow-up. In 4 successful cases Type I AAL, right atrium systolic pressure was reduced after the procedure $(25 \pm 4$ vs. $7 \pm 1 \mathrm{mmHg})$ and NYHA improved $(3.5 \pm 0.6$ vs. $1.0 \pm 0.0)$. In 3 successful Type II patients, the pseudoaneurysm diameter decreased after occlusion $(4.95 \pm 1.8$ vs. $2.0 \pm 1.8 \mathrm{~mm})$. Complete thrombosis in false lumen of stent-segment was observed for all Type III subgroup after the closure. The details are described in the table.

\section{Discussion}

To our best knowledge, our study was the largest case series in one single center for an AAL cohort. Moreover, we employed a new classification system based on the lesion orientation and hemodynamics, which facilitated the interventional strategy. From preliminary result, the overall successful rate was relatively satisfactory.

Hemodynamic instability is frequent in patients with AALs. With this new-defined category, it could be summarized that clinical manifestation of different types of AAL was heterogeneous.

* For a type I AAL, persistent left-to-right shunt caused by Cabrol's shunt technique may lead to a right heart failure. Periprosthetic-to-right atrial fistula (Cabrol's shunt technique) had been used to deal with the catastrophic postoperative hemorrhage in the aortic root and ascending aortic surgery $(15,16)$. However, persistently increasing blood flow of the type I AAL prevented the fistula from closing.

* Type II AAL, as a life-threatening leak often leads to aortic pseudoaneurysm. Due to the persistent leak, pressure of the pseudoaneurysm is relatively high, and thus surrounding organs and tissue are compressed. Rupture is a serious complication, and urgent operation should be performed. In this series, technical and clinical success was $75 \%$ type II AALs. Of note, one failed patient who rejected redo-surgery died of pseudoaneurysm rupture 3 months later. This indicates that Type II AAL should be operated on timely either with interventional procedure or surgical occlusion.

* Most of type III AAL was asymptomatic and often was diagnosed during the post-operative follow-up. It could cause the patency and enlargement of the aortic false lumen.

Redo-surgery is the traditional standard treatment for an AAL, but it is extremely technically challenging with high mortality as it is a large invasive procedure, and thus different percutaneous strategies have been attempted especially for those with high risk for surgery. Hashizume et al. (17) reported endovascular repair of the type II AAL using a stent-graft. As the AAL tends to occur in ascending aorta, approaching aortic arch branches, and therefore landing zone is limited for a stent-graft. Endovascular repair might not be adequate in all such patents. Another study showed a positive result in type II AAL (18) with trans-catheter embolization. However, coil migration might occur in type I or type III AAL since the coil lacks radial force. Accordingly, we attempted to perform a trans-catheter closure of an AAL using different Amplatzer occluders (19,20). 
Our experience demonstrated that careful evaluation of the preferred access was of importance. The femoral artery was commonly used in the present study. For type I or type III AAL, an arteriovenous/true-to-false lumen loop should be built up to facilitate the passage for the delivery of the sheath, and thus femoral vein (for a type I AAL) or a femoral artery with the aortic false lumen involved (for a type III AAL) should be prepared. In this series, the delivery sheath could be only advanced to cross the leak over the loop in a retrograde approach in the type I AAL. The underlying reason might be ascribed to the Dacron patch on the outside of anastomosis restricted the delivery sheath. And the delivery sheath could be advanced over the loop in both the true lumen and the false lumen approach in the type III AAL.

There is no specific device designed for AAL transcatheter closure. Five different Amplatzer occluders were implanted as off-label use in this study: VSO, ADO I, ADO II, AVP II and AVP III. The following points should be considered in use of Amplatzer devices: (I) sufficient distance of the leak in necessary between other important structures such as aortic valve, coronary artery and branches of aortic arch. (II) Because of the AAL is small and inelastic, the size and flexibility of delivery system should be considered. In this study, the failure of delivery systems to be advanced across the leak led to the unsuccessful results. (III) The fabric devices (VSO and ADO I) required large delivery sheaths, which were difficult to cross the leak. Thus, VSO and ADO I were seldom used in our center. The fabric-free occluders (ADO II and AVP family) allow small delivery sheaths; i.e., the maximum diameter of the ADO II is $6 \mathrm{~mm}$, and it is suitable for small AALs. The design of two symmetric disks and a central waist for the ADO II and AVP family is adequate for the deployment in both antegrade and retrograde approaches. AVP II was used in 1 case ascribed to the large central waist and significant elongation as well as device protrusion post-deployment. In recent studies, more AVP III, originally designed for paravalvular leak, was implanted for AALs due to its oblong shape and large ranges of the size options $(21,22)$. (IV) This technique requires a steep learning curve. The procedure is quite complex and time-consuming. Interventional cardiologists should comprehensively understand the design and functions for the different occlusion devices. Various anatomic conditions and surgical types should be precisely planned and dynamic assessment the changes of the anatomy are required during the procedures for the possible alternative intervention.

\section{Study limitations}

Firstly, as a single-center retrospective study of AAL, it was limited by small sample size, however, this was a reasonably large case series. The efficacy of trans-catheter closure was not compared with traditional surgical operation. Such a case-control study will be performed in the future. In addition, long term follow-up is necessary to evaluate the effect.

\section{Conclusions}

Trans-catheter closure appears to be a feasible and reasonable alternative approach for AAL in those highrisk candidates for redo-surgery, and a suggested new classification facilitates the therapeutic strategy. Our preliminary experience warrants further investigations.

\section{Acknowledgments}

Funding: None.

\section{Footnote}

Reporting Checklist: The authors have completed the STROBE reporting checklist. Available at http://dx.doi. org/10.21037/jtd-20-1496

Data Sharing Statement: http://dx.doi.org/10.21037/jtd-201496

Peer Review File: http://dx.doi.org/10.21037/jtd-20-1496

Conflicts of Interest: All authors have completed the ICMJE uniform disclosure form (available at http://dx.doi. org/10.21037/jtd-20-1496). The authors have no conflicts of interest to declare.

Ethical Statement: The authors are accountable for all aspects of the work in ensuring that questions related to the accuracy or integrity of any part of the work are appropriately investigated and resolved. The study was conducted in accordance with the Declaration of Helsinki (as revised in 2013). The study was approved by institutional ethics board of Beijing Anzhen Hospital, and informed consent was taken from all the patients.

Open Access Statement: This is an Open Access article 
distributed in accordance with the Creative Commons Attribution-NonCommercial-NoDerivs 4.0 International License (CC BY-NC-ND 4.0), which permits the noncommercial replication and distribution of the article with the strict proviso that no changes or edits are made and the original work is properly cited (including links to both the formal publication through the relevant DOI and the license). See: https://creativecommons.org/licenses/by-nc-nd/4.0/.

\section{References}

1. Luciani N, De Geest R, Lauria G, et al. Late reoperations after acute aortic dissection repair: Single-center experience. Asian Cardiovasc Thorac Ann 2015;23:787-94.

2. Magnan PE, Albertini JN, Bartoli JM, et al. Endovascular treatment of anastomotic false aneurysms of the abdominal aorta. Ann Vasc Surg 2003;17:365-74.

3. Concistrè G, Casali G, Santaniello E, et al. Reoperation after surgical correction of acute type A aortic dissection: risk factor analysis. Ann Thorac Surg 2012;93:450-5.

4. Tanaka H, Okada K, Kawanishi Y, et al. Clinical significance of anastomotic leak in ascending aortic replacement for acute aortic dissection. Interact Cardiovasc Thorac Surg 2009;9:209-12.

5. Immer FF, Hagen U, Berdat PA, et al. Risk factors for secondary dilatation of the aorta after acute type A aortic dissection. Eur J Cardiothorac Surg 2005;27:654-7.

6. Halstead JC, Meier M, Etz C, et al. The fate of the distal aorta after repair of acute type A aortic dissection. J Thorac Cardiovasc Surg 2007;133:127-35.

7. Geirsson A, Bavaria JE, Swarr D, et al. Fate of the residual distal and proximal aorta after acute type a dissection repair using a contemporary surgical reconstruction algorithm. Ann Thorac Surg 2007;84:1955-64.

8. Dougenis D, Daily BB, Kouchoukos NT. Reoperations on the aortic root and ascending aorta. Ann Thorac Surg 1997;64:986-92.

9. Mohammadi S, Bonnet N, Leprince P, et al. Reoperation for false aneurysm of the ascending aorta after its prosthetic replacement: surgical strategy. Ann Thorac Surg 2005;79:147-52; discussion 152.

10. Kobuch R, Hilker M, Rupprecht L, et al. Late reoperations after repaired acute type A aortic dissection. J Thorac Cardiovasc Surg 2012;144:300-7.

11. Malvindi PG, van Putte BP, Sonker U, et al. Reoperation after acute type A aortic dissection repair: a series of 1004 patients. Ann Thorac Surg 2013;95:922-7.
12. Gendi SM, Kenny D, Hibbeln J, et al. Closure of ascending aorta pseudoaneurysm to right atrium fistula. JACC Cardiovasc Interv 2013;6:418-9.

13. Patel AV, Gupta S, Laffin LJ, et al. One size does not fit all: case report of two percutaneous closures of aortic pseudoaneurysm and review of the literature. Cardiovasc Revasc Med 2014;15:160-4.

14. Shreenivas SS, Lilly S, Desai ND, et al. Percutaneous Closure of an Aortic Pseudoaneurysm Due to Saphenous Vein Graft Dehiscence with an Amplatzer Vascular Plug. JACC Cardiovasc Interv 2013;6:1103-4.

15. Cabrol C, Pavie A, Mesnildrey P, et al. Long-term results with total replacement of the ascending aorta and reimplantation of the coronary arteries. J Thorac Cardiovasc Surg 1986;91:17-25.

16. Panos AL, Suarez M, Salerno TA, et al. Unusual Modification of the Cabrol Shunt for Control of Hemorrhage in Acute Type A Aortic Dissection. J Card Surg 2009;24:544-6.

17. Hashizume K, Shimizu H, Koizumi K, et al. Endovascular aneurysm repair using the periscope graft technique for thoracic aortic anastomotic pseudoaneurysm. Interact Cardiovasc Thorac Surg 2013;16:553-5.

18. Nørgaard A, Andersen LI, Haahr PE, et al. Coil embolization of an anastomotic leak after ascending aorta replacement. Acta Radiol 2008;49:510-2.

19. Pu J, Huang L, Wu W. Trans-catheter Closure of Anastomotic Leakage After Aortic Surgery for Type A Dissection with the Amplatzer Duct Occluder II. Cardiovasc Intervent Radiol 2017;40:1274-7.

20. Pu J, Ke Y, Huang L, et al. Trans-catheter Closure of Aortic Anastomosis Leak Resulting in Patent Cabrol Shunt After Aortic Replacement. JACC Cardiovasc Interv 2017;10:2126-8.

21. García E, Sandoval J, Unzue L, et al. Paravalvular leaks: mechanisms, diagnosis and management. EuroIntervention 2012;supple Q:Q41-52.

22. Cruz-Gonzales, Rama-Merchan JC, Arribas-Jimenez A, et al. Paravalvular Leak Closure With the Amplatzer Vascular Plug III Device: Immediate and Short-term Results. Rev Esp Cardiol (Engl Ed) 2014;67:608-61.

Cite this article as: $\mathrm{Wu} \mathrm{W}, \mathrm{Ke} \mathrm{Y}$, Zhao $\mathrm{H}$, Huang $\mathrm{L}, \mathrm{Pu}$ J. Trans-catheter closure of aortic anastomosis leak after aortic replacement: classifications and techniques. J Thorac Dis 2020;12(9):4883-4891. doi: 10.21037/jtd-20-1496 